\title{
Verified combination of numerical weather- and avalanche- prediction models at Kootenay Pass, British Columbia, Canada
}

\author{
Glaudia ROEGER, ${ }^{1}$ David M. MaGlunG,${ }^{2}$ Roland STUlL ${ }^{3}$ \\ ${ }^{1}$ Pfarrweg 3, D-83043 Bad Aibling, Germany \\ E-mail.croeger@canoemail.com \\ ${ }^{2}$ Department of Geography, University of British Columbia, 1984 West Mall, Vancouver, British Columbia V6T 1Z2, Canada \\ ${ }^{3}$ Atmospheric Science Programme, Department of Earth and Ocean Sciences, University of British Columbia,Vancouver, \\ British Columbia V6T 124, Canada
}

\begin{abstract}
The combination of numerical weather prediction (NWP) and snow avalanche forecasting has been performed using the output of two weather models run at the University of British Columbia, Canada, and a local numerical avalanche-forecasting model developed for Kootenay Pass (McClung and Tweedy, 1994). The main motivations for this work are. (1) to extend the lead time of avalanche forecasts by using NWP forecasts of meteorological variables as input to statistical avalanche-threat models (instead of the traditional method of using current/past observed meteorological variables as input); and (2) to create another tool to help avalanche forecasters in their daily decision-making by making true forecasts instead of "nowcasts". Therefore, verified weather-forecast model output was used as input for the local avalanche-forecasting model at Kootenay Pass. The resulting 24 hour avalanche forecast was compared to observed avalanche occurrences and to the 12 hour avalanche forecast with current weather observations. As a result, the avalanche-model output for the test runs with numerically predicted weather data is comparable in accuracy to the runs with observed weather data. The results also suggest that avalanches may be predicted statistically for 24 hours into the future when high-resolution NWP is used as input, weather- and avalanche-forecast errors taken into account during operational use.
\end{abstract}

\section{INTRODUGTION}

The idea that predictive science can simplify the process of making environmental decisions is deeply problematic in practice, since it brings together a mix of strongly conflicting interests and values (Sarewitz and others, 2000). Predicting complex natural systems (like weather phenomena and snow-avalanche occurrence) has immense theoretical and technical difficulties and there are large uncertainties associated with such predictions.

The motivation for this study was to develop methods to help people make decisions based on environmental predictions, i.e. snow-avalanche forecasters deciding whether to close a highway section or a ski run, whether to trigger avalanches artificially, and what precautions to take with predicted snowfall, which can have important economic consequences. Avalanche forecasting is only one of many applications of weather predictions that require high accuracy. Uncertainties are found by evaluating forecast output against observed data.

Avalanche prediction is a problem of high uncertainty, because avalanches occur over a wide range of conditions and weather prediction is needed to make an avalanche forecast. Many different parameters influence snowpack instability. Most of these variables vary markedly over both time and space.

The goal of snow-avalanche forecasting, defined as the prediction of current and future snow instability in space and time relative to a given triggering level (McClung, 2000), is to minimize the uncertainty about instability introduced by the temporal and spatial variability of the snow cover (including terrain influences), any incremental changes in snow and weather conditions and any variations in human perception. Although experienced avalanche forecasters are skilled in evaluating current conditions and making short-time forecasts (given the expected weather conditions), modern forecasting is moving away from conventional methods (unassisted by computers) toward partially computerized systems (McClung and Schaerer, 1993). Computer-aided avalanche forecasting has three advantages: (1) objectivity, (2) handling large organized datasets, and (3) helping people with limited field experience in their decision-making process (even though the computer models developed up to this point should not be used by people without field experience (McClung, 1995b)). Numerical avalanche prediction refers to organization of a database of previously measured parameters, including avalanche occurrences, for use with a computer to help compare current conditions with past ones. The main emphasis is on meteorological data (McClung and Schaerer, 1993). Software developments range from simple databases to numerical avalanche-forecasting models (AFMs).

Snow-avalanche forecasting is a multi-scale problem (LaChapelle, 1980; McClung and Schaerer, 1993; McClung, 2000), ranging from entire mountain ranges to local slopes. Due to the great variety of climate zones in Canada, the 
Table 1. Variables used for AFM. D: directly gained from NWP; E: empirically estimated

\begin{tabular}{lc}
\hline Parameter for numerical avalanche prediction & Source \\
\hline Weight new snow $(\mathrm{g})$ & $\mathrm{D}$ \\
Height new snow $\mathrm{HN}(\mathrm{cm})$ & $\mathrm{E}$ \\
Water equivalent new-snow HNW $(\mathrm{mm})$ & $\mathrm{D}$ \\
New-snow density $\rho\left(\mathrm{kg} \mathrm{m}^{-3}\right)$ & $\mathrm{E}$ \\
Snow depth $\mathrm{HS}(\mathrm{cm})$ & $\mathrm{E}$ \\
Storm total $(\mathrm{cm})$ & $\mathrm{E}$ \\
Snowfall rate $\left(\mathrm{cm} \mathrm{h}^{-1}\right)$ & $\mathrm{E}$ \\
Ram penetration $\mathrm{PR}(\mathrm{cm})$ & $\mathrm{E}$ \\
Wind speed $\left(\mathrm{km} \mathrm{h}^{-1}\right)$ & $\mathrm{D}$ \\
Wind direction $\left({ }^{\circ}\right)$ & $\mathrm{D}$ \\
Maximum air temperature $\left({ }^{\circ} \mathrm{C}\right)$ & $\mathrm{D}$ \\
Present air temperature $\left({ }^{\circ} \mathrm{C}\right)$ & $\mathrm{D}$ \\
Minimum air temperature $\left({ }^{\circ} \mathrm{C}\right)$ & $\mathrm{D}$ \\
Trend maximum air temperature $\left({ }^{\circ} \mathrm{C}\right)$ & $\mathrm{D}$ \\
Trend present air temperature $\left({ }^{\circ} \mathrm{C}\right)$ & $\mathrm{D}$ \\
\hline
\end{tabular}

demand for numerical avalanche prediction is at the mesoscale, which implies more accurate prediction than for synoptic-scale forecasts (McClung, 1995a). The combination of weather forecast and avalanche prediction requires the combination of two different scales, which seriously limits on the accuracy of avalanche forecasting due to extrapolations and simplifications (Föhn, 1998).

The comparison of output variables from numerical weather prediction (NWP) models and input variables for AFMs shows that many of the NWP variables can be directly applied into an AFM or can easily be derived. The remaining AFM variables (usually measured in the field and not directly received from weather forecasts) can be estimated or approximated with empirical relationships. When weather forecasts are reasonably accurate on the local scale and they are included in AFMs, the two fields may be combined successfully, allowing the prediction of future instabilities and hence, avalanches.

McClung and Tweedy (1994) developed a numerical AFM, which is used operationally at Kootenay Pass, Canada, out to 12 hours into the future, based on current observations. With meteorological forecast data that are sufficiently accurate, it might be possible to predict avalanches out to 24 hours into the future. The AFM features parametric discriminant analysis using Bayesian statistics to predict avalanche occurrences. Cluster techniques are then employed in multidimensional discriminant space to analyze avalanche occurrences by the method of nearest neighbors. The model calculates the probability of overall avalanche occurrence and avalanche type (dry or wet). In addition, the 30 nearest neighbors to the data input, together with the magnitude and frequency of avalanching (avalanche-activity index (AAI); see section 2) and the Mahalanobis distance, are displayed. Detailed information such as avalanche location, size and trigger mechanism is available for each neighbor. Both output avalanche probability and nearest neighbors are analyzed with the methods described in section 4.

Two forecast output datasets have been used as input for the AFM: (1) Mesoscale Compressible Community (MC2) $2 \mathrm{~km} 24$ hour forecast, and (2) combination Non-hydrostatic Modeling System (NMS) $10 \mathrm{~km}$ and MC2 $2 \mathrm{~km} 24$ hour forecast. These forecast outputs have been proven to show the best overall results for Kootenay Pass, which was determined by weather-forecast verification (Roeger and others, 2001, 2003; see section 4). This true avalanche forecast for 24 hours was tested against observed avalanche occurrence and against the avalanche "nowcast" with current weather observations.

The idea of developing separate models for predictions of physical processes was formed long ago. A model chain of weather data, snowpack structure and avalanche-risk forecasting has been developed in France (Giraud and others, 1998). The combination of actual weather-forecast data from high-resolution NWP models and numerical avalanche prediction has now been carried out in Canada with the tools available for the specific problems here.

\section{GHARACTERISTICS OF VARIABLES IN AVALANCHE FORECASTING}

The variables used in the AFM developed by McClung and Tweedy (1994) are given in Table 1. These variables have been determined using significance tests (McClung and Tweedy, 1993). Significant variables have a high correlation with the AAI, which is the sum of avalanche sizes recorded in each time period. It is a simple index of the magnitude and frequency of avalanching within a time period. Table 1 indicates whether the value for this variable can be directly obtained from weather-forecast output (indicated with D) or if it has to be estimated with numerical, empirical methods (E), as described below and in section 3c.

All empirically estimated variables in Table 1 depend on new-snow density $\rho$. Therefore, a comprehensive analysis of new-snow density was performed to determine $\rho$ from meteorological data (section 3c). The height of new snow HN was then calculated:

$$
\mathrm{HNW}=\frac{\rho \mathrm{HN}}{\mathrm{a} \rho_{\mathrm{L}}},
$$

where $\mathrm{HNW}$ is the equivalent liquid-water depth of the new snow (given as part of the standard weather-forecast output), $\rho_{\mathrm{L}}=1000 \mathrm{~kg} \mathrm{~m}^{-3}$ is the density of liquid water, and $a=0.1\left(\mathrm{~cm} \mathrm{~mm}^{-1}\right)$ is used to convert between the typical units used for snow and liquid-water depth (see Table 1).

With the value for $\mathrm{HN}(\mathrm{cm})$, snowfall rate was calculated as an average rate since the last observation 6 or 18 hours). The total snow depth HS and the ram penetration depth PR were estimated with the observed snow depth or ram penetration, respectively, from the last observation plus the new-snow height HN. Ram penetration is a measure of vertical depth of penetration into the snow with a standard ram penetrometer (CAA, 1995).

Storm total was calculated by adding up the estimated new-snow height since the beginning of the storm. The weight of new snow is calculated from the predicted water equivalent new snow HNW ( $\mathrm{mm}$ ) using the density for water $\left(1000 \mathrm{~kg} \mathrm{~m}^{-3}\right)$. The water equivalent of new snow is predicted by the weather-forecast models.

Potential rain instead of snow as precipitation is recognized by the avalanche model through temperature $\left(>0^{\circ} \mathrm{C}\right)$, and height of new snow being equal to zero $(\mathrm{HN}=0)$ in combination with the water equivalent of new snow not being zero $(\mathrm{HNW} \neq 0)$. 


\section{DATA}

\section{a. Meteorological forecasts}

The two research NWP models used here were the MC2 version 4.8 model, refined by Recherche en Prévision $\mathrm{Nu}-$ mérique (RPN) in Canada, and the University of Wisconsin (U.S.A.) NMS. Both were run real-time for this verification study, making daily forecasts on multiple grids out to 48 hours into the future. To simulate operational forecast conditions, no manual tuning was done after the forecast. Both models and their verification results are described in detail in Roeger and others (in press).

The MC2 model (Benoit and others, 1997) was run with horizontal gridpoint spacing of 90,30, 10, 3.3 and $2 \mathrm{~km}$. The NMS model was developed primarily by G. Tripoli at the University of Wisconsin (Tripoli, 1992), and is run at the University of British Columbia for two-way interactive nests with 90,30 and $10 \mathrm{~km}$ grid spacing. For each weather station, forecast values from the surrounding four or nine gridpoints were interpolated to calculate the forecast for the exact location. For the verification, the forecasts were divided into two forecast time periods: $0-24$ and 24-48 hours into the future.

\section{b. Snow and weather observations}

The highway operation Kootenay Pass $\left(49.05^{\circ} \mathrm{N}, 117.0^{\circ} \mathrm{W}\right)$, in the southern Selkirk Mountains of southeastern British Columbia, consists of two weather stations for collecting manual and remote data: Kootenay Pass and Stagleap. The manual observation site at the summit of Kootenay Pass is located at $1780 \mathrm{~m}$ a.s.l. in an open area surrounded by trees. It is fairly sheltered, so wind observations may be biased. Precipitation measurements are expected to be representative for the area, and temperatures are typical for this elevation. Temperature is measured at shelter height $(2 \mathrm{~m})$ above the ground or snow surface. Stagleap is a remote weather station at the top of a ridge (2140 $\mathrm{m}$ a.s.l.) and well exposed to the wind. Therefore, wind speeds are typical for this mountain ridge and elevation, but are not representative for some avalanche starting zones at mid-mountain elevation, especially on the lee side. At this station, winds are measured remotely (anemometer) atop a $10 \mathrm{~m}$ high tower. Data are gathered according to the guidelines from the Canadian Avalanche Association (CAA, 1995). The morning observation is between 0500 and $0800 \mathrm{~h}$ (local time), the afternoon observation between 1500 and $1700 \mathrm{~h}$. The observation periods thus differ in length. They have been approximated into categories of 6 and 9 hours (morning to afternoon) and 15 and 18 hours (afternoon to next morning).

Measurement problems, especially related to winter weather (e.g. stalled anemometers due to significant riming effects), may occur at all weather stations. At Kootenay Pass, information about the working condition of the instruments and a first check of the measured value (within a certain range depending on the variable) is obtained automatically with the measurements (true/false signal). For this project, all measured data were again examined in detail and only data that were correctly measured (within the range of uncertainty related to the measurement itself) were retained for verification.

Precipitation rate was relatively complicated to assess. Observed precipitation data at Kootenay Pass were collected hourly in $\mathrm{mm} \mathrm{h}^{-1}$ from gauge measurements (remote observations). Solid precipitation (snow) was measured with snow measurement boards (CAA, 1995), and total precipitation (solid and liquid) with a precipitation gauge (both manually twice a day). These manual observations include total snow height on the ground (HS), snowfall during the last 9 and 18 hours using the standard observation board, and 24 hours using the new-snow observation board. Snowfall during the last storm event is measured with the storm observation board. All observations are measured according to the CAA guidelines (CAA, 1995).

The forecast from both NWP models was given in $\mathrm{mm}$ (or mm w.e., respectively) as 3 hour total (sum of solid and liquid) and 3 hour solid precipitation. Total precipitation was verified by readings from the precipitation gauge at Kootenay Pass. Three 1 hour remote measurements were summed to a 3 hour value for comparison to the 3 hour forecast. Solid precipitation was compared with the 9 or 18 hour measurements from the standard observation board; 3 hour forecasts were added up to 9 or 18 hours.

\section{c. New-snow density analysis}

In order to estimate new-snow density and new-snow height in $\mathrm{cm}$ - when the water equivalent of new snow is given in $\mathrm{mm}$ - it was necessary to analyze the characteristics of new-snow density as a function of air temperature and wind speed.

Similar analyses have been performed in the European Alps by Pahaut (1975) and Meister (1985). Meister suggests a quasi-linear relationship or a power function for density dependent on air temperature. For the quasi-linear relationship, he separates air temperature into ranges $<-1^{\circ} \mathrm{C}$ and $\geq-1{ }^{\circ} \mathrm{C}$. Pahaut (1975) found a function for new-snow density, which includes a linear dependence of temperature and a square-root function for wind.

We considered several relationships between new-snow density and temperature. A detailed description of the analysis can be found in Roeger (2001). We found that newsnow density does not depend on wind speed but depends significantly on air temperature at the Kootenay Pass study plot. The relation is optimal when air temperature is averaged with the former observation in a 2:1 ratio (e.g. Meister, 1985). The best fit for new-snow density based on averaged air temperature was an exponential function as in Equation (2). The values for the constants $b, m$ and $c$ are 130, 1.2 and 30, respectively. The Pearson correlation coefficient $(r=0.59)$ and standard error $(\mathrm{ME}=0.34)$ suggest that the new-snow density is only approximate.

$$
\rho=b\left(m^{T}+c\right) .
$$

\section{AVALANCHE-FOREGAST VERIFICATION METHODS}

The AFM (McClung and Tweedy, 1994) was run with three different weather-data sources. First, the input came from weather observations equivalent to the operational use of the avalanche model at Kootenay Pass. Second, it was run with weather input from the forecast output of the MC2 $2 \mathrm{~km}$-grid 24 hour forecast for all parameters and their derived parameters as described in section 2. Third, input data were combined from the output of the MC2 and NMS models, in order to find optimal values for the numerical avalanche model. For this combination, the MC2 $2 \mathrm{~km}$-grid 24 hour forecast gave temperatures and winds, and the 
Table 2. Contingency table definition, where $A-D$ are the counts of events in each category, out of $\mathcal{N}$ total events

\begin{tabular}{llll}
\hline & & \multicolumn{2}{c}{ Observation } \\
& & Yes & No \\
\hline \multirow{3}{*}{ Forecast } & Yes & A & B \\
& No & C & D \\
\hline
\end{tabular}

NMS 24 hour forecast gave precipitation (water equivalent new snow). Since density is estimated based on air temperature, these values come from the MC2 model as well, but calibrated from records at Kootenay Pass. All input parameters that are based on both density and precipitation are therefore derived using a combination of both weather models.

The AFM has an option to allow the forecaster to specify an a priori probability for avalanche occurrence and avalanche type, but this was not used. Instead, the a priori probabilities were kept at the default value of 0.5 for all three test scenarios so that the results are easily comparable. Also, this choice allows the model prediction of avalanche type (dry or wet) to be unbiased.

Two methods of comparison were used when different input data were tested. First, each run was tested against observed avalanche data. The second method was a comparison of model output statistics of the different input datasets.

\section{a. Verification with avalanche observation data}

Verification of model results against observed avalanche data was done with contingency table analysis to assess accuracy (see Table 2, illustrating a $2 \times 2$ contingency table). Our measurements include the hit rate $(H)$, the percentage of forecasts correct (PFG), the probability of detection (POD), the false-alarm ratio (FAR) and the bias ratio (BIAS). These quantities are given as Equations (A.1-A.5) in the Appendix.

The hit rate (or the percentage of forecasts correct) is the ratio of correct forecast events to the total number of events. The lowest (worst) possible hit rate is zero. A value of 1 would represent a perfect forecast.

The bias ratio is the comparison of the average forecast with the average observation. It is the ratio of the "yes" forecasts to the number of "yes" observations. The value $\mathrm{BIAS}=1$ indicates that the event was forecast correctly the same number of times that it was observed. Bias ratios $>1$ indicate that the event was forecast more often than it was observed (over-forecasting). Conversely, bias ratios $<1$ indicate under-forecasting. The bias ratio is not an accuracy measure because it contains no information about the correspondence between the forecasts and observations of the event on particular occasions (Wilks, 1995).

Equations (A.6) and (A.7) show the Heidke skill score (HSS) and the true skill score (TSS). They are derived by contingency table analysis as well.

The Heidke skill score is based on the hit rate as the basic accuracy measure, but also takes into account the random nature of forecasts. The hit rate expected for random forecasts is taken as the reference accuracy measure. Forecasts equivalent to the reference forecasts receive zero scores.
Table 3. Results from contingency table analysis for avalanche moisture type (Fig. 1) (obs: observed data; fcst: forecast)

\begin{tabular}{lcccc}
\hline AFMinput & PFG & Bias ratio(wet) & TSS & HSS \\
& $\%$ & & & \\
\hline Weather obs & 92 & 1.25 & 0.89 & 0.83 \\
MC2 fcst & 80 & 1.67 & 0.71 & 0.60 \\
MC2 + NMS fcst & 85 & 1.50 & 0.78 & 0.68 \\
\hline
\end{tabular}

Negative scores represent forecasts that are worse than the reference forecasts. Perfect forecasts receive a Heidke score of 1 (Wilks, 1995).

TSS is a measure of true forecast skill. In short, the true skill score is the POD, adjusted by the POFD (probability of false detection). It was originally proposed by Peirce (1884), then known as the Hanssen-Kuipers discriminant or Kuipers' performance index (Murphy and Daan, 1985), or referred to as the true skill score as discussed in Flueck (1987) (Wilks, 1995). It is similar to the Heidke skill score, but the random forecast that is taken into account is constrained to be unbiased. A value of 1 represents a perfect forecast, 0 is random/neutral, and TSS $<0$ are inferior to a random forecast.

The categorial variables in the contingency table analysis are moisture type of avalanche (dry-wet), avalanche event prediction, and avalanche event prediction separated into dry and wet avalanches.

For the avalanche moisture type, the observed avalanche type was compared with the predicted moisture type. Therefore, the total number $\mathcal{N}$ is fairly small, since only days with avalanche occurrence could be used.

Avalanche events are predicted (i.e. the model output is an avalanche warning) when the probability of dry avalanching is $\geq 0.6$ and the probability of moist-wet avalanching is $\geq 0.7$, based on the empirical results of McClung and Tweedy (1994). This was tested for all avalanches as well as for dry and wet avalanches separately.

\section{b. Comparison of model-output statistics}

The model output was analyzed (1) statistically by using the results of cluster analysis, and (2) with general numerical indicators of similar performance. Cluster analysis gives the fraction of nearest neighbors with avalanches (historical data) in the first 10 or 30 neighbors, respectively. A description of the method of nearest neighbors can be found in McClung and Tweedy (1994).

Numerical indicators of similar performance are the numbers of same nearest neighbors in the first 10 and 30, the magnitude of avalanching of the nearest neighbors (AAI) and the relative distance of neighbors 1,10 and 30 to the day predicted. The magnitude of each day is the AAI defined by McClung and Tweedy (1994) as the sum of avalanche sizes using the Canadian size classification system (McClung and Schaerer, 1993, p. 252).

\section{RESULTS}

\section{a. Verification with avalanche observation data}

For avalanche moisture type (i.e. the model predicts the type of avalanche likely to occur), the run with observed 


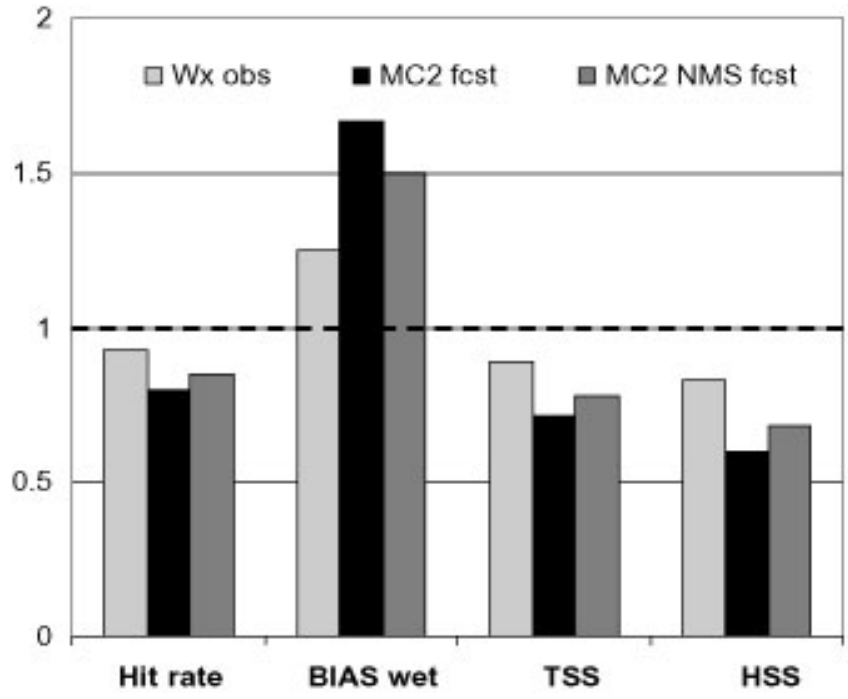

Fig. 1. Results from contingency table analysis for avalanche moisture type, prediction vs observation, Kootenay Pass, Fanuary-April 2000. A value of 1.0 is best in all categories.

weather data (Wx obs) achieves the best results in all categories (Table 3). Figure 1 shows a histogram of results from contingency table analysis. The run with combined data from MC2 and NMS gives better results than the run with MC2 forecast data in all categories. A bias can be seen towards wet avalanches, which are over-forecast with all three input sets. This means that the avalanche model misclassified some dry avalanches as wet avalanches. For the hit rate and the skill scores (TSS and HSS), the runs with forecast data produce slightly lower values than the run with observation data.

Figure 2 shows results for overall avalanche occurrence. Values are listed in Table 4. Here, the MC2 model achieves the best results in all categories. The test run with $\mathrm{MC} 2+\mathrm{NMS}$ model output also has better results than the run with weather observations, except for one category (POD). The PFC is $61 \%$ with the MC2 model. The MC2NMS combination achieves $52 \%$ whereas the measured weather observations achieve only $38 \%$. The latter value is lower than the success rate reported by McClung and Tweedy (1994), who used Bayesian statistics in order to allow forecaster judgment to enter. All three datasets over-forecast avalanche occurrence (bias ratio $>1$ ). The FAR is 0.77 from weather observations, 0.73 from the combined MC2-NMS

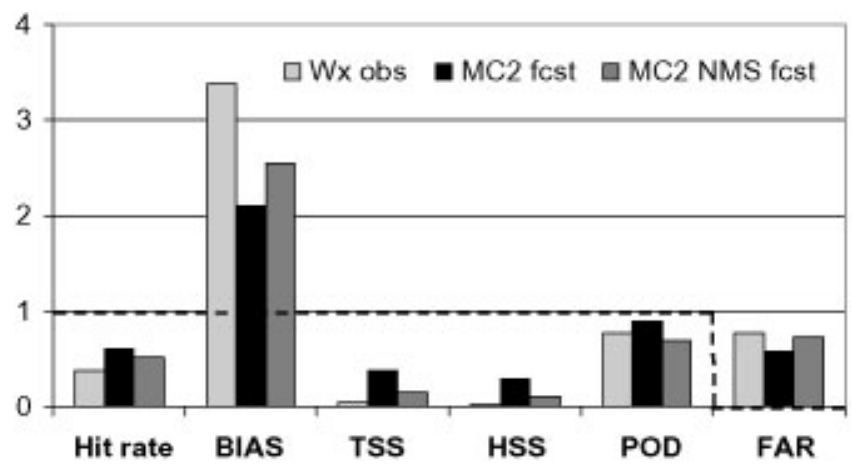

Fig. 2. Results from contingency table analysis for overall avalanche occurrence (wet and dry avalanching), Kootenay Pass, January-April 2000. Perfect forecasts achieve zero for FAR and 1.0 in all other categories.
Table 4. Results from contingency table analysis for avalanche prediction (wet and dry avalanching) (Fig. 2) (obs: observed data; fcst: forecast)

\begin{tabular}{lcccccc}
\hline AFMinput & PFC & Bias ratio & TSS & HSS & POD & FAR \\
& $\%$ & & & & & \\
\hline Weather obs & 38 & 3.38 & 0.05 & 0.02 & 0.77 & 0.77 \\
MC2 fcst & 61 & 2.10 & 0.38 & 0.29 & 0.90 & 0.57 \\
MC2 + NMS fcst & 52 & 2.54 & 0.16 & 0.10 & 0.69 & 0.73 \\
\hline
\end{tabular}

forecast and 0.57 from the MC2 forecast. All three input sets show low skill statistics (HSS and TSS). However, the run with MC2 model output achieves much better values than the other two test scenarios.

Separation into dry and wet avalanches improves the hit rate. For dry avalanches (contingency table results are shown in Figure 3 and listed in Table 5), the run with MC2 model input achieves $85 \%$ PFC, a very good result. It is almost unbiased towards over-forecasting dry avalanches (bias ratio $=1.14)$ and has a relatively low FAR (0.38). It has significantly higher hit-rate and skill scores than the results from weather observations and the MC2-NMS combined forecast. Only for POD does the run with weather observations achieve a higher value $(0.89$ compared to 0.71 with the MC2 model). The MC2-NMS combination is slightly better than the weather observation output, except for POD. Its hit rate is 0.69 (compared to a value of 0.52 achieved by the run with observation data).

The results for wet avalanches (Fig. 4; Table 6) do not so clearly favor one type of input. The run with observed weather data has the highest hit rate (0.85) and the lowest bias ratio (with over-forecasting of wet avalanches by slightly more than $100 \%$ ). But it also shows the lowest skill score values and POD.

The MC2 model performs worst for hit rate $(0.70)$ and bias ratio (4.33), but has relatively high skill and POD. The test run with MC2 + NMS model input mostly shows average performance. Its hit rate is 0.83 .

\section{b. Comparison of model output statistics}

Figure 5 shows results from cluster analysis: median values and upper and lower quartile of the difference between the AFM output with weather observation input and the AFM

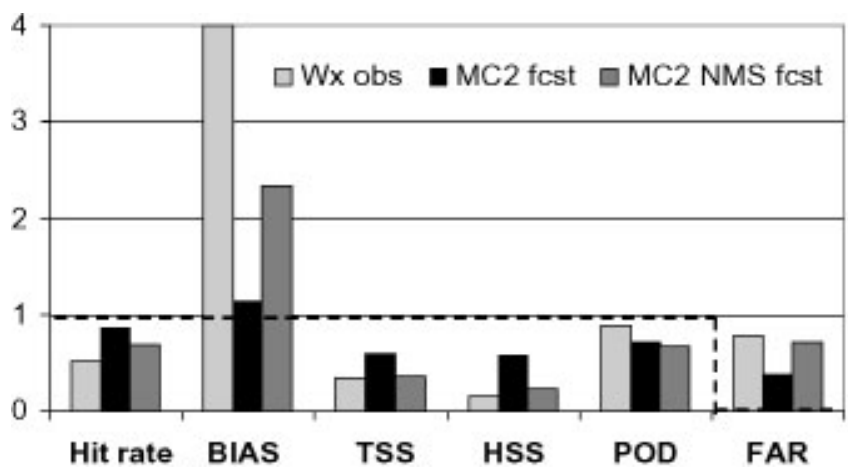

Fig. 3. Results from contingency table analysis for dry avalanches, Kootenay Pass, January-April 2000. Perfect forecasts achieve zero for FAR and 1.0 in all other categories. 
Table 5. Results from contingency table analysis for dry avalanche prediction (Fig. 3) (obs: observed data; fcst: forecast)

\begin{tabular}{lcccccc}
\hline AFMinput & PFC & Biasratio & TSS & HSS & POD & FAR \\
& $\%$ & & & & & \\
\hline Weather obs & 52 & 4 & 0.34 & 0.15 & 0.89 & 0.78 \\
MC2 fcst & 85 & 1.14 & 0.60 & 0.57 & 0.71 & 0.38 \\
MC2 + NMS fcst & 69 & 2.33 & 0.36 & 0.23 & 0.67 & 0.71 \\
\hline
\end{tabular}

Table 6. Results from contingency table analysis for wet avalanche prediction (Fig.4) (obs: observed data; fcst: forecast)

\begin{tabular}{lcccccc}
\hline AFMinput & PFG & Bias ratio & TSS & HSS & POD & FAR \\
& $\%$ & & & & & \\
\hline Weather obs & 85 & 2.25 & 0.38 & 0.24 & 0.50 & 0.78 \\
MC2 fcst & 70 & 4.33 & 0.67 & 0.27 & 1.00 & 0.77 \\
MC2 +NMS fcst & 83 & 3.00 & 0.58 & 0.30 & 0.75 & 0.75 \\
\hline
\end{tabular}

output with weather-forecast model input. Ideally, the median difference in avalanche occurrence should be $0 \%$ with minimized upper and lower quartile. The median difference of $0 \%$ is achieved from both test runs except for the 30 day results with MC2 input. The spread of the difference is plotted as the interquartile range (IQR; line that combines the upper and lower quartile). The values for IQR should be small, but are about as large as, or even larger than, the median values of the avalanche occurrence for each run (given in Table 7).

The MC2 median value of -3 (30 nearest neighbors) shows that the avalanche model predicts, on average, one more nearest neighbor with avalanches when MC2 weather-forecast data are used as input imposed to measured weather-input data. The error of $20 \%$ in the direction of forecasting more nearest neighbors with avalanches (lower quartile: $-20 \%$ ) underlines this general trend of the MC2 input dataset. The MC2-NMS combination shows the opposite trend. The error for forecasting fewer days with avalanches (upper quartile) is $20 \%$ and $13 \%$ for the first 10 and 30 neighbors, respectively. This trend of the MC2-NMS combination could be hazardous in operational use if the error is not accounted for.

Table 7. Values according to Figure 5: upper and lower quartile and IQR of difference to AFM-weather observation combination and median values of avalanche occurrence (\%) for each AFM-weather-forecast combination, 10 or 30 nearest neighbors (nn)

\begin{tabular}{|c|c|c|c|c|}
\hline & \multicolumn{2}{|c|}{$\begin{array}{c}M C 2 \\
\text { fcst input }\end{array}$} & \multicolumn{2}{|c|}{$\begin{array}{c}M C 2+\mathcal{N} M S \\
\text { fcst input }\end{array}$} \\
\hline & $10 \mathrm{nn}$ & $30 n n$ & $10 \mathrm{nn}$ & $30 \mathrm{nn}$ \\
\hline Median difference $(\%)$ & 0 & -3 & 0 & 0 \\
\hline Upper quartile of difference & 10 & 3 & 20 & 13 \\
\hline Lower quartile of difference & -20 & -20 & -10 & -7 \\
\hline IQR difference & 30 & 23 & 30 & 20 \\
\hline Median of avalanche occurrence & 24.9 & 25.6 & 19.0 & 19.6 \\
\hline
\end{tabular}

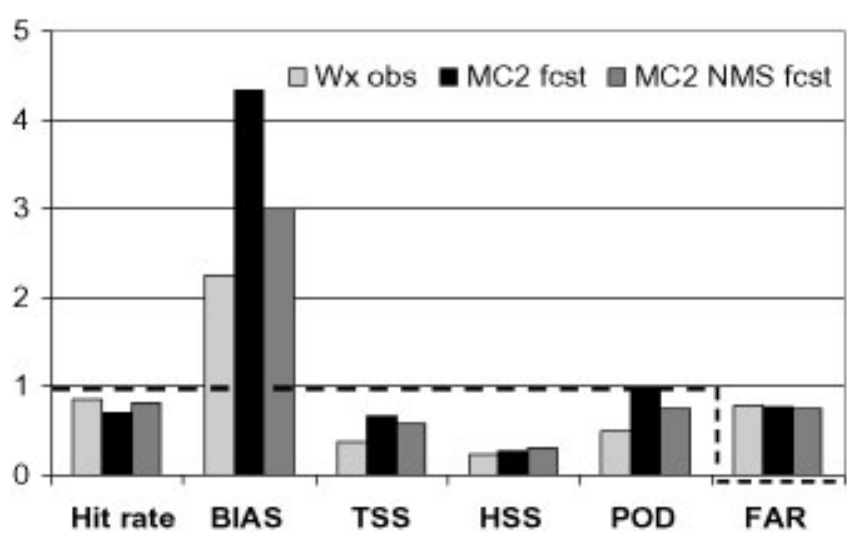

Fig. 4. Results from contingency table analysis for wet avalanches, Kootenay Pass, January-April 2000. Perfect forecasts achieve zero for FAR and 1.0 in all other categories.

The historical database of the AFM contains 2138 weather and avalanche observation datasets. It is therefore fairly good when the same nearest neighbors (same days/ same observations) are shown for the observation and forecast mode. For the MC2 input dataset, $49 \%$ of the test runs give at least 1 common nearest neighbor out of 30 , and $27 \%$ of the first 10. The MC2-NMS combination achieves $51 \%$ and $23 \%$ of 30 and 10 nearest neighbors, respectively.

Figure 6 and Table 8 give the difference to weather observation input data of the average AAI per day for 10 and 30 nearest neighbors, respectively, which represents the magnitude of avalanching. Again, the median difference should be zero, which is only achieved by the MC2 input dataset of the 10 nearest neighbors. The MC2-NMS combination shows a trend towards under-forecasting the average avalanche activity, which is shown by the positive median value and higher absolute values of the upper quartile compared to the lower quartile. This should be carefully considered when the avalanche model is used operationally. The mean error of 2.1 and 1.5 for the first 10 and all 30 nearest neighbors, respectively, is relatively high, compared to the mean value for AAI (averaged per day) of the 71 analyzed days: 1.8.

Figure 7 shows the comparison of the relative distance

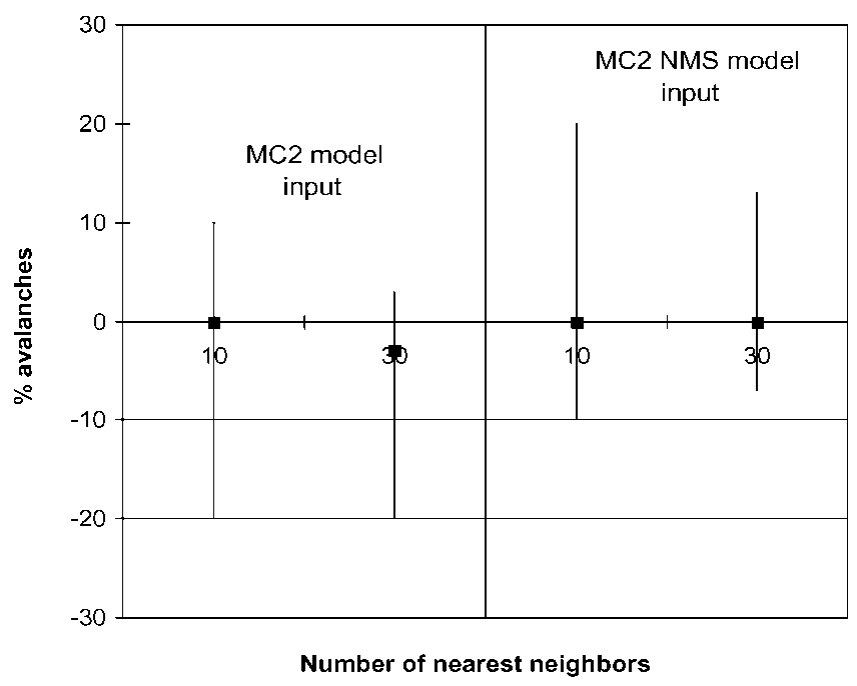

Fig. 5. Results from cluster analysis: neighbors with avalanche occurrence in \%. Difference to results with weather observation input. Median, lower and upper quartile. A value of zero is best. 


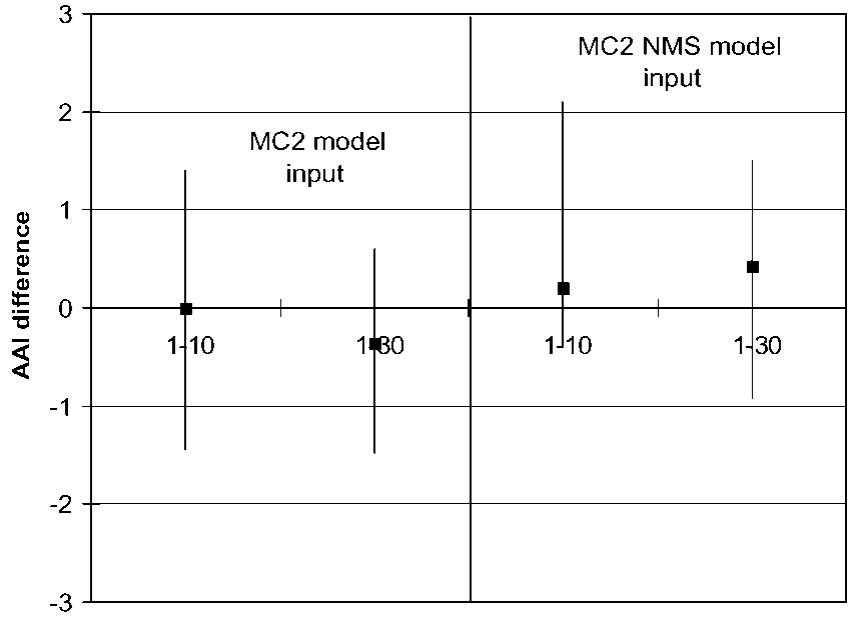

Number of nearest neighbors

Fig. 6. Average AAI per day: difference to results with weather observation input. Median, lower and upper quartile. A value of zero is best.

(Mahalanobis distance) to each nearest neighbor. This distance from both forecast combinations is increasingly larger with higher nearest neighbors than the distance calculated with weather observation. For neighbor 1, the difference in relative distance as well as the IQR from both forecast combinations is fairly small, which represents a good result (values are listed in Table 9). The MC2 input shows higher median differences and spread than the MC2-NMS combination, which suggests that the results of the latter are closer to the weather observation dataset.

\section{SUMMARY AND GONGLUSIONS}

Input datasets from two numerical weather-forecast realtime models at the University of British Columbia and measured weather data were combined with a local AFM running at Kootenay Pass. Output from the MC2 2 km grid 24 hour forecast for all parameters and their estimations was chosen for the first test run for the numerical AFM.

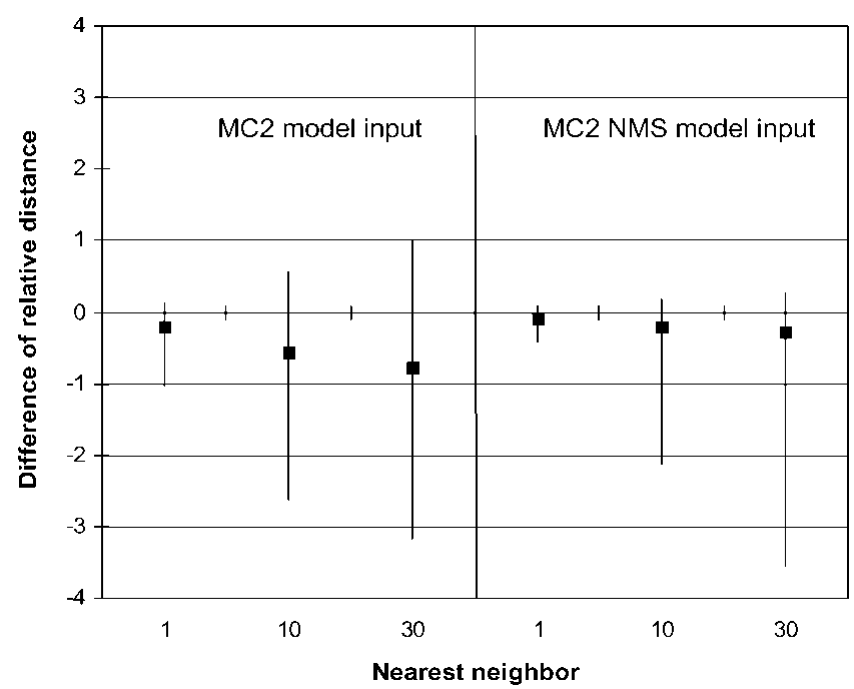

Fig. 7. Relative distance at neighbors 1, 10 and 30: difference to results with weather observation input. Median, lower and upper quartile. A value of zero is best.
Table 8. Values according to Figure 6: median, upper and lower quartile and IQR of difference to AFM-weather observation combination of AAI for each AFM-weather-forecast combination, 10 or 30 nearest neighbors ( $n n$ )

\begin{tabular}{lcccc}
\hline & \multicolumn{2}{c}{$M C 2$} & \multicolumn{2}{c}{$M C 2+\mathcal{N}$ NS } \\
& \multicolumn{2}{c}{ fcst input } & \multicolumn{2}{c}{ fcst input } \\
& $10 \mathrm{nn}$ & $30 \mathrm{nn}$ & $10 \mathrm{nn}$ & $30 \mathrm{nn}$ \\
\hline Median difference & 0 & -0.35 & 0.2 & 0.43 \\
Upper quartile of difference & 1.4 & 0.6 & 2.1 & 1.5 \\
Lower quartile of difference & -1.45 & -1.48 & -0.4 & -0.92 \\
IQR difference & 2.85 & 2.08 & 2.5 & 2.42 \\
\hline
\end{tabular}

For the second test run with the AFM, input data were combined from the output of two forecast models. for this second case, the MC2 $2 \mathrm{~km}$ grid 24 hour forecast gave temperatures and winds, and the NMS 24 hour forecast gave precipitation (water equivalent new snow), which has fairly good results for this parameter.

The combination of NWP and numerical avalanche forecasting gave promising results. The AFM output for the model runs with numerically predicted weather data is very similar to the run with observed weather data. All three datasets over-forecast avalanche occurrence, which means that avalanche warnings are issued too often. The MC2 trial achieved the best results in all categories for overall avalanche occurrence. The large values for false-alarm rates are potentially troublesome for the operational use of the model since they tend to cause avalanche warnings to be disbelieved. The median differences of cluster-analysis results between the test runs with weather-forecast input and the run with weather observations are ideal with $0 \%$ (except for one case). The MC2 dataset is more conservative than the MC2-NMS combination. It shows not only more nearest neighbors with avalanches than the weather observation data, but also a higher AAI on average. The MC2-NMS combination shows a trend towards lower average AAI and fewer nearest neighbors with avalanches than the weather observation data. Hence, the MC2 seems to be better for operational use, but the mean error should be accounted for as well. Relative distances suggest that the MC2-NMS results are closer to the weather observation results than the results with the MC2.

This combination and its verification results indicate that avalanches may be statistically predicted out to 24 hours into the future with high-resolution NWP as input.

Table 9. Values according to Figure 7: median, upper and lower quartile and IQR of difference to AFM-weather observation combination of relative distance at neighbors 1, 10, and 30 for each AFM-weather-forecast combination

\begin{tabular}{lrrrrrrr}
\hline & \multicolumn{3}{c}{ MC2 } & \multicolumn{4}{c}{ MC2 + NMS } \\
& \multicolumn{2}{c}{$\begin{array}{c}\text { fcst input } \\
\end{array}$} & \multicolumn{4}{c}{$f$ cst input } \\
& 10 & 30 & 1 & 10 & 30 \\
\hline Median difference & -0.19 & -0.56 & -0.77 & -0.08 & -0.19 & -0.27 \\
Upper quartile of difference & 0.12 & 0.55 & 1.01 & 0.09 & 0.17 & 0.28 \\
Lower quartile of difference & -1.03 & -2.63 & -3.18 & -0.41 & -2.13 & -3.56 \\
IQR difference & 1.15 & 3.18 & 4.19 & 0.5 & 2.3 & 3.84 \\
\hline
\end{tabular}


However, the weather-forecast errors should be taken into account during operational use.

Overall, these results show that the accuracy of the AFM with weather-forecast data input is in the same order as with measured weather data input, and short-time high-resolution NWP output is already sufficiently accurate to be used for operational avalanche forecasting. But it should be kept in mind that data from only one season have been tested so far. The next step would be to test the model in daily use with short-time NWP as input for several seasons, in order to find out how the model can be used operationally.

\section{ACKNOWLEDGEMENTS}

This research was sponsored by Canadian Mountain Holidays, the Natural Sciences and Engineering Research Council of Canada, Forest Renewal BC, Environment Canada and BC Hydro. C. Roeger was supported by the German Academic Exchange Service (DAAD). The measured weather data for this study were provided by the Ministry of Transportation and Highways of British Columbia. The research coordination and infrastructure was managed by the Geophysical Disaster Computational Fluid Dynamics Centre. We are extremely grateful for the support of all these organizations.

\section{REFERENCES}

Benoit, R., M. Desgagné, P. Pellerin, S. Pellerin, Y. Chartier and S. Desjardins. 1997. The Canadian MC2: a semi-Lagrangian, semi-implicit wideband atmospheric model suited for finescale process studies and simulation. Monthly Weather Review, 125(10), 2382-2415.

Canadian Avalanche Association (CAA). 1995. Observation guidelines and recording standards for weather, snowpack and avalanches. Revelstoke, B.C., Canadian Avalanche Association.

Flueck, J. A. 1987. A study of some measure of forecast verification. In 10th Conference on Probability and Statistics in Atmospheric Sciences, October 6-81987, Edmonton, Alberta, Canada. Proceedings. Boston, MA, American Meteorological Society.
Föhn, P. M. B. 1998. An overview of avalanche forecasting models and methods. In Hestnes, E., ed. 25 Years of Snow Avalanche Research, Voss, 12-16 May 1998. Proceedings. Oslo, Norwegian Geotechnical Institute, 19-27.

Giraud, G., E. Brun, Y. Durand and E. Martin. 1998. Safran/Crocus/Mépra models as a helping tool for avalanche forecasters. In Hestnes, E., ed. 25 Years of Snow Avalanche Research, Voss, 12-16 May 1998. Proceedings. Oslo, Norwegian Geotechnical Institute, 108-112.

LaChapelle, E. R. 1980. The fundamental processes in conventional avalanche forecasting. 7. Glaciol., 26(94), 75-84.

McClung, D. M. 1995a. Computer assistance in avalanche forecasting. In ISSW'94. International Snow Science Workshop, 30 October-3 November 1994, Snowbird, Utah. Proceedings. Snowbird, UT, P.O. Box 49, 310-313.

McClung, D. M. 1995b. Use of expert knowledge in avalanche forecasting. Def. Sci. 7., 45(2), 117-123.

McClung, D. M. 2000. Predictions in avalanche forecasting. Ann. Glaciol., 31, 377-381.

McClung, D. M. and P. A. Schaerer. 1993. The avalanche handbook. Seattle, WA, The Mountaineers.

McClung, D. M. and J. Tweedy. 1993. Characteristics of avalanching. Kootenay Pass, British Columbia, Canada. F. Glaciol., 39(132), 316-322.

McClung, D. M. and J. Tweedy. 1994. Numerical avalanche prediction. Kootenay Pass, British Columbia, Canada. f. Glaciol., 40(135), 350-358.

Meister, R. 1985. Density of new snow and its dependence on air temperature and wind. Zürcher Geogr. Schr. 23, 73-79.

Murphy, A. H. and H. Daan. 1985. Forecast evaluation. In Murphy, A. H. and R.W. Katz, eds. Probability, statistics, and decision making in the atmospheric sciences. Boulder, CO, Westview Press, Inc.

Pahaut, E. 1975. Les cristaux de neige et leurs métamorphoses. Saint-Martind'Hères, Météo-France. Centre d'Études de la Neige. (Monographie de la Météorologie Nationale 96.)

Peirce, C. S. 1884. The numerical measure of the success of predictions Science, 4, 453-454.

Roeger, C. 2001. Verification of numerical weather prediction and avalanche forecasting. (M.Sc. thesis, University of British Columbia.)

Roeger, C., D. McClung, R. Stull, J. Hacker and H. Modzelewski. 2001. A verification of numerical weather forecasts for avalanche prediction. Cold Reg. Sci. Technol., 33(2-3), 189-205.

Roeger, C., R. Stull, D. McClung, J. Hacker, H. Modzelewski and X. Deng. 2003. Verification of mesoscale numerical weather forecasts in mountainous terrain for application to avalanche prediction. Weather and Forecasting., 18 (6) 1140-1160.

Sarewitz, D., R. A. Pielke, Jr and R. Byerly, Jr. 2000. Prediction, science, decision-making and the future of nature. Washington, DC, Island Press.

Tripoli, G. J. 1992. A nonhydrostatic mesoscale model designed to simulate scale interaction. Monthly Weather Review, 120(7), 1342-1359.

Wilks, D. S. 1995. Statistical methods in the atmospheric sciences. New York, Academic Press. (International Geophysics Series 59.)

\section{APPENDIX}

\section{EQUATIONS FOR CONTINGENGY TABLE ANALYSIS}

$\begin{array}{rrr}\text { Measurement } & \text { Equation } & \text { Range } \\ \text { Ferfect } & \text { forecast }\end{array}$

\begin{tabular}{|c|c|c|c|c|}
\hline Hit rate $H$ & $H=\frac{A+D}{N}$ & $0-1$ & 1 & (A.1.1) \\
\hline Percentage of forecast correct PFC & $\mathrm{PFC}=H(100 \%)$ & $0-100 \%$ & $100 \%$ & (A.1.2) \\
\hline Threat score TS & $\mathrm{TS}=\frac{A}{A+B+C}$ & $0-1$ & 1 & (A.2) \\
\hline Probability of detection POD & $\mathrm{POD}=\frac{A}{A+C}$ & $0-1$ & 1 & (A.3) \\
\hline False-alarm ratio FAR & $\mathrm{FAR}=\frac{B}{A+B}$ & $0-1$ & 0 & (A.4) \\
\hline Bias ratio & $\mathrm{BIAS}=\frac{A+B}{A+C}$ & $\infty$ to $+\infty$ & 0 & (A.5) \\
\hline Heidke skill score HSS & $\mathrm{HSS}=\frac{2(A D-B C)}{(A+C)(C+D)+(A+B)(B+D)}$ & -1 to +1 & 1 & (A.6) \\
\hline True skill score TSS & $\mathrm{TSS}=\frac{A D-B C}{(A+C)(B+D)}=A+C-\frac{B}{B+D}$ & -1 to +1 & 1 & (A.7) \\
\hline
\end{tabular}

